\title{
Accuracy of direct magnetic resonance imaging-guided placement of drug infusion cannulae
}

\author{
Prashant Chittiboina, MD, ${ }^{1}$ John D. Heiss, MD, ${ }^{1}$ and Russell R. Lonser, MD ${ }^{1,2}$ \\ ${ }^{1}$ Surgical Neurology Branch, National Institute of Neurological Disorders and Stroke, National Institutes of Health, Bethesda, \\ Maryland; and ${ }^{2}$ Department of Neurological Surgery, The Ohio State University Wexner Medical Center, Columbus, Ohio
}

\begin{abstract}
An intraoperative MRI (iMRI)-compatible system has been developed for direct placement of convection-enhanced delivery (CED) cannulae using real-time imaging. To establish the precision and feasibility of this technology, the authors analyzed findings in patients who underwent direct iMRI CED cannula placement.

Three consecutive patients underwent iMRI-guided placement of CED infusion cannulae ( 6 cannulae) for treatment of diffuse intrinsic brainstem glioma (2 patients) or Parkinson's disease (1 patient). Convective infusion cannulae were guided to the target using the ClearPoint iMRI-based navigation platform (MRI Interventions, Inc.). Placement accuracy was analyzed.

Real-time iMRI during infusion cannula insertion allowed for monitoring of trajectory accuracy during placement. During cannula insertion, no reinsertions or changes due to errors in targeting were necessary. The mean radial error was $1.0 \pm 0.5 \mathrm{~mm}( \pm \mathrm{SD})$. There was no correlation between the total length of the planned trajectory and the radial error (Pearson's coefficient: $-0.40 ; p=0.5$ ). The mean anteroposterior and lateral errors were $0.9 \pm 0.5$ and $0.3 \pm 0.2 \mathrm{~mm}$, respectively. The mean in-plane distance error was $1.0 \pm 0.4 \mathrm{~mm}$. The mean tip error (scalar distance between the planned target and actual tip) was $1.9 \pm 0.9 \mathrm{~mm}$. There was no correlation between the length of the planned trajectory and any of the measured errors. No complications were associated with cannula placement.

Real-time iMRI-based targeting and monitoring of infusion cannula placement is a safe, effective, and accurate technique that should enable more selective perfusion of brain regions.

http://thejns.org/doi/abs/10.3171/2014.11.JNS131888
\end{abstract}

KEY WORDS accuracy; convection-enhanced delivery; brainstem glioma; gadolinium; magnetic resonance imaging; targeting; surgical technique

$\mathrm{C}$ ONVECTION-ENHANCED delivery (CED) is used to directly deliver putative therapeutic agents to the central nervous system., $2,67,18$ This delivery method utilizes fluid convection, which is bulk flow of infusate from a single point source (infusion cannula tip) into the surrounding extracellular spaces, driven by a small hydrostatic pressure gradient. ${ }^{17}$ Consequently, accurate placement of infusion cannulae is critical to precisely place the infusion point source in a location that ensures optimum distribution of the infusate. ${ }^{19}$ Specifically, emerging data indicate that it is critical that the infusion cannulae be placed with a high degree of accuracy to selectively and completely perfuse diseased deep brain anatomical re- gions (including nuclear and subnuclear targets) for maximal pharmacological effect. .11,14, $^{19}$

To avoid accuracy errors associated with conventional stereotaxy and to provide real-time feedback during cannula placement, intraoperative MRI (iMRI) technology has been developed that enables 3D targeting and placement of infusion cannulae during contemporaneous imaging. Originally called "prospective stereotaxy," the method was first used to perform brain biopsies of deep brain structures with real-time iMRI. ${ }^{12}$ The method has been adapted to achieve submillimeter accuracy with real-time high-field iMRI for insertion of deep brain stimulation (DBS) electrodes. ${ }^{4,10}$ The current iMRI platform uses in-

ABBREVIATIONS AAV2-GDNF = adeno-associated virus encoding glial cell line-derived neurotrophic factor; CED = convection-enhanced delivery; DBS = deep brain stimulation; DIPG = diffuse intrinsic pontine glioma; IL13-PE = interleukin-13-Pseudomonas exotoxin; iMRI = intraoperative MRI; PD = Parkinson's disease.

SUBMITTED September 3, 2013. ACCEPTED November 24, 2014.

INCLUDE WHEN CITING Published online January 16, 2015; DOI: 10.3171/2014.11.JNS131888.

DISCLOSURE This study was funded by the intramural research program of the National Institute of Neurological Disorders and Stroke (NINDS) at the National Institutes of Health. IL13-PE was provided by InSys Therapeutics, Inc., under a cooperative research and development agreement with NINDS. The SmartFlow cannula and ClearPoint system were provided by MRI Interventions, Inc., under a clinical trial agreement with NINDS. 
traoperative imaging to progressively align a guide frame with the target and to assess the postimplantation position of infusion cannulae relative to the target.

While this infusion cannula placement paradigm has been described in nonhuman primates, ${ }^{13,14}$ it had not been applied clinically. We describe and analyze the application of this technology in patients undergoing convective drug delivery for diffuse intrinsic pontine glioma (DIPG) and Parkinson's disease (PD).

\section{Methods \\ Patients}

The combined Neuroscience Institutional Review Board of the National Institutes of Health approved this study. Two consecutive pediatric patients enrolled in a Phase 1, open-label, dose-escalation study of interleukin 13-Pseudomonas exotoxin (IL13-PE) coinfused with GdDTPA were included. The mean age of pediatric patients undergoing CED for DIPG was 10.5 years (ages 5 and 16 years). One adult patient enrolled in a Phase 1, open-label, dose escalation study of adeno-associated virus encoding glial cell line-derived neurotrophic factor (AAV2-GDNF) coinfused with Gd-DTPA in PD was included. The age of this patient was 67 years. Informed consent was obtained from the legal guardians of pediatric patients with DIPG and from the adult patient with PD.

\section{System Components}

MRI-guided stereotactic CED procedures were performed using the MRI-compatible ClearPoint system (MRI Interventions, Inc.). This system includes an infusion cannula (SmartFlow), SmartFrame MRI-guided trajectory frame, and SmartGrid MRI-guided planning grid. The ClearPoint Workstation is a dedicated laptop computer that uses proprietary software to communicate with the MRI console. The surgeon uses the workstation for planning and target accuracy assessment. The SmartFrame MRI-guided trajectory frame contains a guide frame for the cannula that houses an MRI-visible fluid stem (Gd-impregnated). The frame contains fiducials that are detected when MR images are obtained with the SmartFrame attached. The procedure is performed within an iMRI suite (MR scanner: 1.5-T Achieva, Philips).

\section{Accuracy Estimation}

Accuracy of insertion was analyzed by comparing the position of the actual cannula tip with that of the planned insertion point (Fig. 1). Because the distal $18 \mathrm{~mm}$ of the cannula is not covered by ceramic and is not visible on MR images, the actual cannula tip position was determined by a line projecting $18 \mathrm{~mm}$ from the end of the MRI-visible ceramic component of the cannula. The location of the actual cannula tip was confirmed at infusion initiation when imaging revealed contrast emanating from the actual cannula tip. Error measurements between the planned target and actual cannula tip were obtained in all 3 planes. Errors are reported in terms of actual tip error (scalar distance between the planned target and actual cannula tip) and in-plane distance (the distance between the planned target and target plane projection in the plane of the planned target if the actual cannula tip were in the plane of the planned target [i.e., vertical distance between the planned target and actual cannula tip $=0 \mathrm{~mm}]$ ). The primary measure for error reporting was the radial distance (radial distance: the scalar distance between the planned target and closest point on the actual trajectory).

\section{Statistical Analysis}

Error measurements were tested for linear correlation by calculating the Pearson's coefficient and using a 1-tailed test for significance. For testing the relationship of errors to the sagittal and coronal angle, 2-tailed Spearman correlation was used.

\section{Technique \\ Patient Preparation}

General anesthesia is administered. Two MRI surface coils are placed on each side of the head with head clamp pins through the bore of the coils ("Princess Leia" configuration), and the patient's head is fixed in an MRIcompatible 3-pin head clamp, which is attached to the MR scanner table top. The table enters the MR scanner bore, placing the patient's head at the isocenter of the MR scanner bore. Survey images are acquired. The patient is moved further through the MR scanner bore until the head is surgically accessible at the back of the scanner. The right and/or left frontal region is then prepared and draped. An accordion drape is fixed to the front and back of the scanner to allow movement of the patient's head from the back end of the scanner to the isocenter of the bore, while maintaining sterility.

\section{Planning}

A localizing grid is placed over the scalp, and a preliminary volumetric scan is obtained. The anterior commissure, posterior commissure, and midsagittal plane are identified on imaging. An entry point, target, and surgical trajectory are chosen using the proprietary software. SmartGrid coordinates for the target are established. A parasagittal linear incision is made at the determined site. The SmartFrame aiming device is secured to the outer table of the skull. A volumetric image is obtained for adjusting the trajectory and to evaluate brain shift after bur hole placement (Fig. 2).

\section{Navigation and Cannula Insertion}

The location of the guide frame for the cannula within the aiming device is calculated on the workstation. With the patient at isocenter in the MR scanner, adjustments to the frame are made by changing the pitch, roll, and offset adjustment wheels on the guide frame using a hand controller that extends from the Smart Frame to the back of the scanner (Fig. 3). MRI scans are obtained after each adjustment and until the guide frame is aligned with the anatomical target.

The cannula is then advanced through the guide frame to its final target. Images are acquired to evaluate the trajectory during insertion to allow for trajectory revision. Before starting infusion, another MR image is obtained to confirm the trajectory and actual cannula tip position (Fig. 


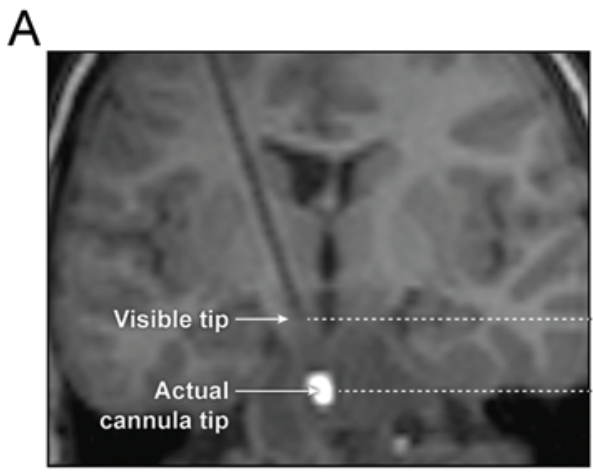

C

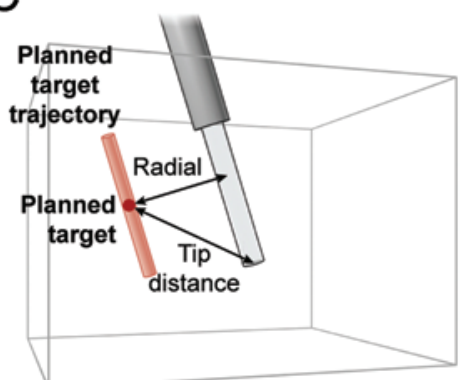

B

D

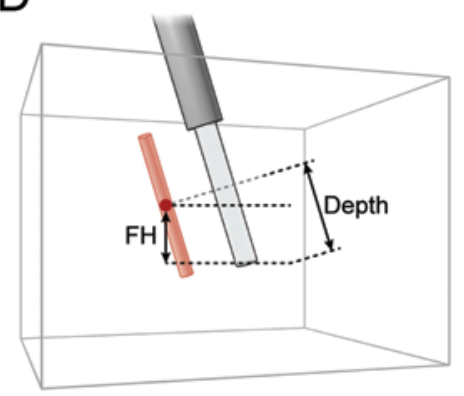

E

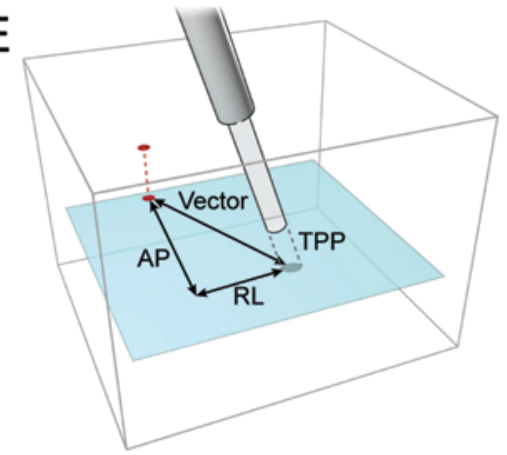

FIG. 1. A graphical depiction of the errors. A and B: The location of the visible tip at the distal end of the ceramic cannula body. The silica inner lumen is not visible on MRI but is visualized at the start of the infusion. C-E: The radial distance is the closest distance of the planned target to the actual cannula $(\mathrm{C})$ and tip distance is the scalar distance between the actual catheter tip and planned target. The foot-head $(\mathrm{FH})$ distance is the vertical distance between the planes of the actual tip and the planned target (D). The anteroposterior (AP) and right-left (RL) or lateral distance is calculated in the target plane from the target plane projection (TPP) of the catheter (E).

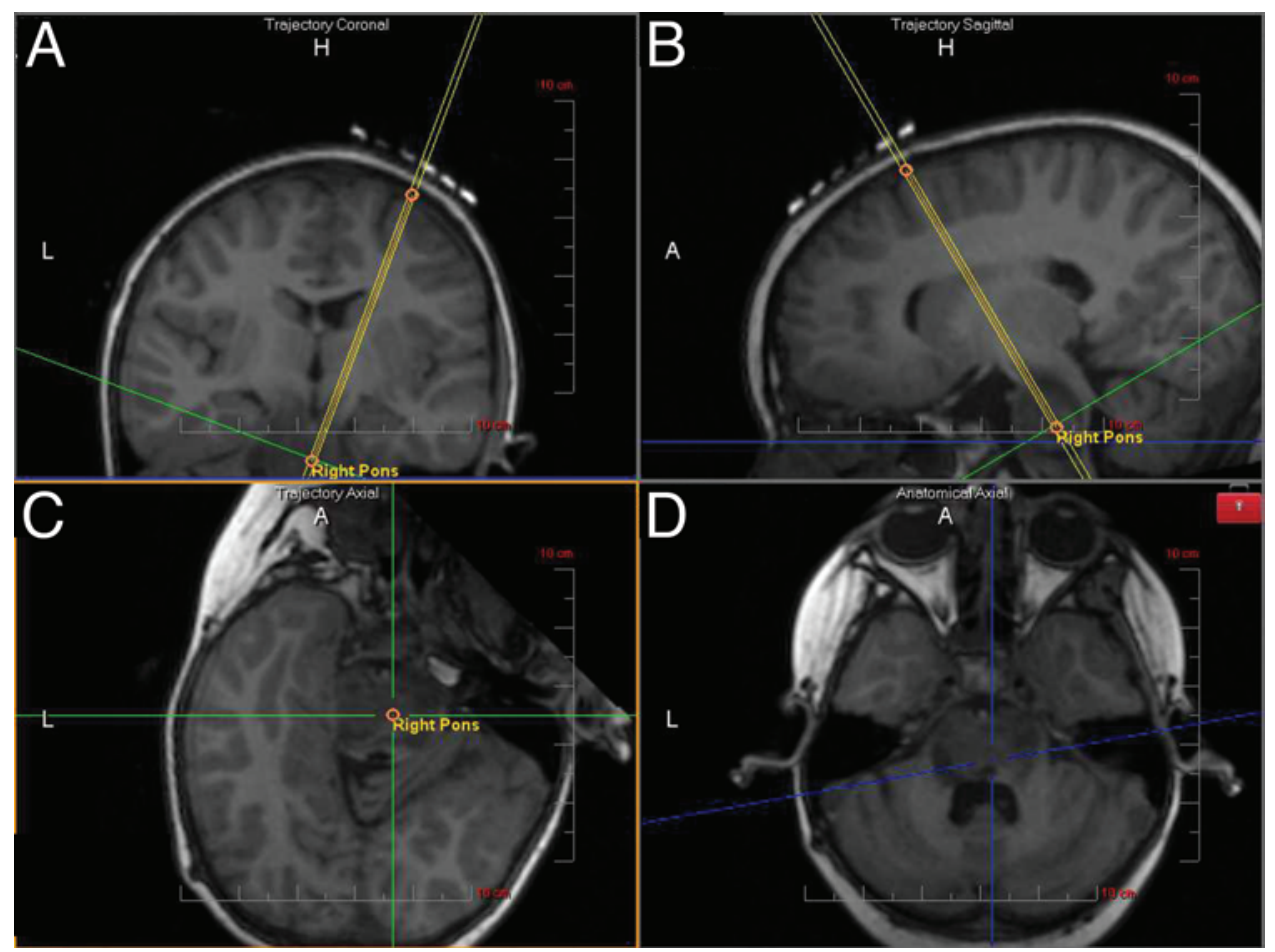

FIG. 2. Using the known entry and target points, the planned trajectory is calculated by the software. In this step, the trajectory is denoted by double yellow lines. A and B: Coronal (A) and sagittal $(B)$ trajectory views (parallel to the planned trajectory). C and D: The plane orthogonal to the trajectory (trajectory axial; $C$ ) and the anatomical axial (D) views. $A=$ anterior; $H=$ head (superior); $L=$ left. 

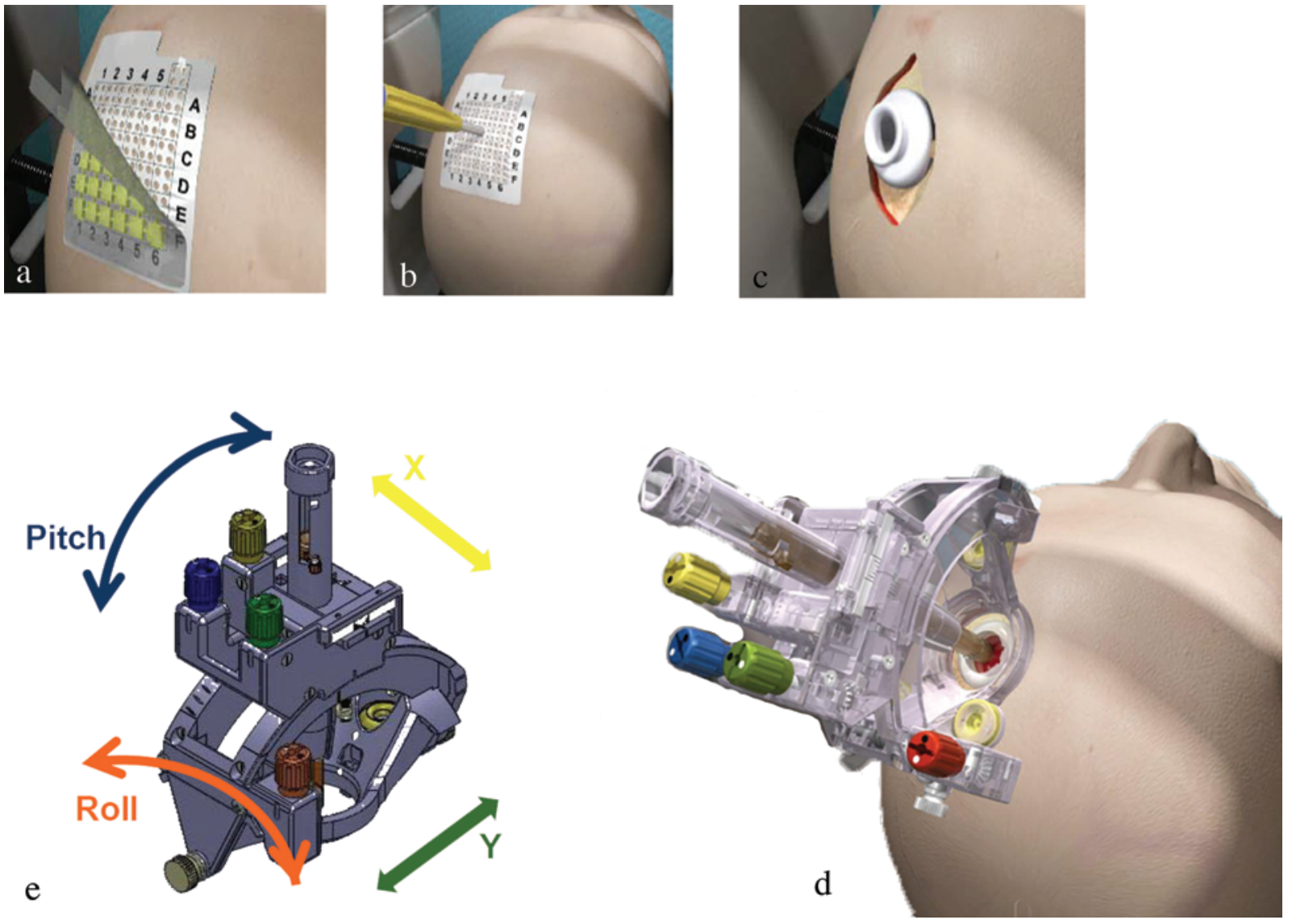

FIG. 3. The outer layer of the grid is peeled off (a), and a mark is made with an augur at the selected site (b). A bur hole is made after making a parasagittal incision (c). The SmartFrame is then attached to the outer table (d). Large adjustments are made with pitch and roll controls. Fine adjustments are made with $\mathrm{x}$ - and $\mathrm{y}$-axis offset controls (e). Images courtesy MRI Interventions, Inc., Memphis, TN. Published with permission.

1). The infusion cannula is connected to the pump, and infusion is started. For multiple insertions on the ipsilateral side, the guide frame was readjusted to the new target without detaching it from the outer table of the skull.

\section{Cannula Design}

The MRI-compatible cannula consists of a multistep tip design to minimize backflow during CED. The working length of the cannula is $27.2 \mathrm{~cm}$, with a rigid ceramic body extending $25.4 \mathrm{~cm}$. The distal $18 \mathrm{~mm}$ of the cannula is not visible on MR images. The silica inner lumen extends out unprotected for $3 \mathrm{~mm}$ and has an outer diameter of $0.665 \mathrm{~mm}$. The inner diameter of the cannula is $200 \mu \mathrm{m}$ throughout the total length of the cannula, resulting in a total priming volume of $0.04 \mathrm{ml}$.

\section{Infusate Characteristics}

IL13-PE38QQR (or IL13-PE: Cintredekin besudotox, IL-13-Pseudomonas exotoxin chimeric protein cytotoxin; NeoPharm, Inc.) infusate is a recombinant chimeric cytotoxin consisting of the enzymatically active portion of Pseudomonas exotoxin A (PE38, the cytotoxic agent) and human interleukin 13 (IL-13, the tumor-targeting molecule). The AAV2-GDNF infusate consists of an AAV2 virus containing human GDNF complementary DNA, human cytolomegalovirus promoter, and 3' UTR (untranslated region) sequences (supplied by the University of California, San Francisco). A total of $450 \mu \mathrm{l}$ AAV2-GDNF infusate is delivered to each side: $300 \mu \mathrm{l}$ per anterior putamen and $150 \mu \mathrm{l}$ per posterior putamen. Both infusates are coinfused with Gd-DTPA. The Gd-DTPA concentration is $5 \mathrm{mM}$ for IL-13PE coinfusion and $1 \mathrm{mM}$ for AAV2GDNF coinfusion.,14

\section{Convective Infusion}

A Medfusion (Model 3500) syringe infusion pump is used to generate a hydrostatic (convective) pressure during infusion that is transmitted from a therapeutic-filled syringe ( $5 \mathrm{ml}$ total volume) to the inner infusion cannula via polyethylene tubing (inner diameter $0.023 \mathrm{in}$, outer diameter 0.050 in). The infusion cannula is then placed in the targeted site.

\section{Intraoperative MRI}

An initial standard volumetric scan (T1-weighted; TR $16 \mathrm{msec}$, TE $3 \mathrm{msec}$, FOV $240 \mathrm{~mm}$ ) is obtained for initial planning. Once anterior commissure and posterior commissure landmarks are identified, a bur hole is planned 
based on the scalp grid. A second standard volumetric scan is acquired with the guide frame in position over the bur hole. A T1-weighted scan is obtained for targeting (T1weighted 3D turbo spin echo; TR $250 \mathrm{msec}$, TE $66 \mathrm{msec}$, FOV $80 \mathrm{~mm}$ ). After the trajectory is defined, sequential scan plane images are obtained (T1-weighted align scan slab; TR $20 \mathrm{msec}$, TE $3.743 \mathrm{msec}$, FOV $100 \mathrm{~mm}$ ). Following initial roll/pitch adjustments, T1-weighted coronal and sagittal images are acquired for offset adjustment (T1weighted spin echo; TR $300 \mathrm{msec}$, TE $45 \mathrm{msec}$, FOV 240 $\mathrm{mm})$. T2-weighted insertion scans are obtained to evaluate the actual trajectory of the catheter tip (T2 insert stylet scan; TR $2000 \mathrm{msec}$, TE $81 \mathrm{msec}$, FOV $240 \mathrm{~mm}$ ). Final volumetric scans are obtained for error evaluation.

\section{Results}

\section{Cannula Insertion}

Six cannulae were placed in the 3 patients included in this study ( 2 patients with DIPG and 1 patient with PD). In the PD patient, 4 CED cannulae (2 cannulae bilaterally into the putamen) were placed during 1 procedure (Table 1). Real-time MRI was performed during placement of each cannula. This permitted confirmation of an accurate cannula trajectory during placement. Because each cannula followed the planned trajectory, no reinsertion or alterations during placement were required.

\section{Accuracy Measurement}

The mean radial error was $1.0 \pm 0.5 \mathrm{~mm}$. No correlation between the total length of the planned trajectory and the radial error was found (Pearson's coefficient: $r=$ $-0.40 ; \mathrm{p}=0.5)$. The mean tip error was $1.9 \pm 0.9 \mathrm{~mm}$. The anteroposterior and lateral errors were $0.9 \pm 0.5$ and $0.3 \pm 0.2 \mathrm{~mm}$, respectively (errors are calculated in the plane of the planned target). The mean in-plane distance was $1.0 \pm 0.4 \mathrm{~mm}$. The superoinferior (foot-head) error is the distance between the planes of the MRI-visible tip and planned target. No correlation between the length of the planned trajectory and any of the measured errors was found (Pearson's coefficient; tip error: $\mathrm{r}=0.6, \mathrm{p}=0.1$; inplane: $\mathrm{r}=-0.2, \mathrm{p}=0.3)$. No correlation between radial error (or other errors) and age (Pearson's coefficient: $\mathrm{r}=$ $0.5, \mathrm{p}=0.4$ ), sex (Spearman: $\mathrm{r}=0.5, \mathrm{p}=0.7$ ), procedure side $(\mathrm{r}=-0.4, \mathrm{p}=0.1)$, or primary pathology $(\mathrm{r}=0.4, \mathrm{p}=$ $0.7)$ was found. No correlation between the sagittal and coronal angle of the trajectory and the measured errors (radial error: sagittal, $\mathrm{r}=0.03, \mathrm{p}=1.0$; coronal, $\mathrm{r}=-0.09$, $\mathrm{p}=0.8$ ) or in-plane distance was found (sagittal, $\mathrm{r}=-0.2$, $\mathrm{p}=1.0 ;$ coronal, $\mathrm{r}=-0.3, \mathrm{p}=0.7)($ Table 2$)$.

\section{Complications}

No complications were associated with cannula placement, including hemorrhage or infection.

\section{Discussion}

\section{Prior Reports}

Use of this iMRI platform for placement of CED cannulae has been reported in nonhuman primates. ${ }^{16,19}$ In these preclinical feasibility studies, the putamen and thalamus
TABLE 1. Targeting details

\begin{tabular}{|c|c|c|c|c|c|}
\hline $\begin{array}{l}\text { Case } \\
\text { No. }\end{array}$ & $\begin{array}{l}\text { Age } \\
\text { (yrs), } \\
\text { Sex }\end{array}$ & Diagnosis & Target & Entry & $\begin{array}{c}\text { Cannula } \\
\text { Insertion } \\
\text { Time (mins) }\end{array}$ \\
\hline 1 & $16, M$ & DIPG & Right pons & Right frontal & 161 \\
\hline 2 & $5, \mathrm{~F}$ & DIPG & Right pons & Right frontal & 118 \\
\hline \multirow[t]{4}{*}{3} & \multirow[t]{4}{*}{$67, M$} & \multirow[t]{4}{*}{ PD } & $\begin{array}{l}\text { Right anterior } \\
\text { putamen }\end{array}$ & Right frontal & \multirow[t]{4}{*}{ 55/cannula } \\
\hline & & & $\begin{array}{l}\text { Left anterior } \\
\text { putamen }\end{array}$ & Left frontal & \\
\hline & & & $\begin{array}{l}\text { Right posterior } \\
\text { putamen }\end{array}$ & Right frontal & \\
\hline & & & $\begin{array}{l}\text { Left posterior } \\
\text { putamen }\end{array}$ & Left frontal & \\
\hline
\end{tabular}

were selected as target sites, and Gd-DTPA was used as a surrogate imaging marker to evaluate targeting accuracy and volume of distribution of the infusate. These previous studies reported 11 cannula placements with satisfactory insertion on the first attempt in each case. No infusate reflux or distribution beyond the target structure was found. Similar to this current clinical trial, the mean targeting error was $0.8 \mathrm{~mm}$, and the error was independent of depth of target structure from the cortical surface. The authors reported that no catheter-related hemorrhages or other adverse events occurred. ${ }^{16,19}$

The platform used in this study has also been validated for placement of subthalamic nucleus DBS electrodes for PD. Consistent with the accuracy found in the current report, Larson and colleagues ${ }^{10}$ described 53 electrode implantations in 29 patients. These authors found a mean radial error of $1.2 \pm 0.65 \mathrm{~mm}$ and an absolute DBS tip error of $2.2 \pm 0.92 \mathrm{~mm}$ (between planned and actual DBS lead tip placement). When the authors compared their data to historical controls in the literature, they found improved targeting accuracy using the ClearPoint system for DBS lead placement.

\section{Current System \\ General}

We describe the use of the iMRI platform for the delivery of CED infusate to the supratentorial and infratentorial brain structures. Patients in this study underwent convective infusion to the brainstem and putamen. The patient who received infusion of AAV2-GDNF for PD underwent safe and successful targeted placement of multiple cannulae (4 cannulae) during a single setting. One patient underwent a repeat CED procedure performed 6 weeks after the initial procedure. For the second procedure, the same incision and bur hole were used. The planning stage included an entry point and trajectory that were displaced by $1 \mathrm{~mm}$ posterior to the previous cannula tract. Due to differences in planning and the workflow of the second procedure, it was excluded from this study.

\section{Accuracy of the System}

Consistent with preclinical data, we found that the mean radial error for the final catheter position was $1.0 \pm$ 
TABLE 2. Summary of accuracy evaluation

\begin{tabular}{|c|c|c|c|c|c|c|c|c|c|}
\hline $\begin{array}{l}\text { Case } \\
\text { No. }\end{array}$ & Target & $\begin{array}{c}\text { Trajectory } \\
\text { Length (mm) }\end{array}$ & Radial (mm) & $\begin{array}{l}\text { Corrected } \\
\text { Depth (mm) }\end{array}$ & $\begin{array}{l}\text { RL Error } \\
\text { (mm) }\end{array}$ & $\begin{array}{l}\text { AP Error } \\
(\mathrm{mm})\end{array}$ & $\begin{array}{c}\text { In-Plane } \\
\text { Distance } \\
(\mathrm{mm})\end{array}$ & FH Error $(\mathrm{mm})$ & $\begin{array}{l}\text { Tip Error } \\
\text { (mm) }\end{array}$ \\
\hline 1 & Right pons & 119.23 & 1 & -0.3 & 0.7 & 0.8 & 1.1 & -16.8 & 1.04 \\
\hline 2 & Right pons & 105.08 & 0.5 & -1.3 & 0.2 & 0.6 & 0.6 & -16.9 & 1.39 \\
\hline \multirow[t]{4}{*}{3} & Right anterior putamen & 72.18 & 1.7 & -2.3 & 0.2 & 1.5 & 1.5 & -18.9 & 2.86 \\
\hline & Left anterior putamen & 75.19 & 1 & -0.9 & 0.3 & 0.9 & 0.9 & -18.1 & 1.35 \\
\hline & Right posterior putamen & 72.92 & 1.3 & -0.9 & 0.1 & 1.3 & 1.3 & -18.2 & 1.58 \\
\hline & Left posterior putamen & 77.17 & 0.5 & 3.2 & 0.4 & 0.1 & 0.5 & -10.6 & 3.24 \\
\hline \multicolumn{2}{|c|}{ Summary statistics (mean \pm SD) } & $86.96 \pm 20.10$ & $1.00 \pm 0.46$ & $-0.42 \pm 1.89$ & $0.32 \pm 0.21$ & $0.87 \pm 0.50$ & $0.98 \pm 0.39$ & $-16.58 \pm 3.04$ & $1.91 \pm 0.90$ \\
\hline
\end{tabular}

$0.5 \mathrm{~mm}$. This and other error measurements indicated that the iMRI platform had sufficient accuracy to successfully place the tip of the infusion cannula to the planned target repeatedly. There was no correlation of the errors with depth, age, or diagnosis. During a preliminary evaluation of the iMRI system for DBS lead placement, small anterior $(0.5 \pm 0.3 \mathrm{~mm})$ and lateral $(0.4 \pm 0.3 \mathrm{~mm})$ errors were reported by Larson and colleagues..$^{10}$ Similarly, we found anterior $(0.9 \pm 0.5 \mathrm{~mm})$ and lateral $(0.3 \pm 0.2 \mathrm{~mm})$ biases in the system. Previously, these biases were attributed to slight nonlinearities in the MR space. ${ }^{10}$ Further investigation with larger studies of CED catheter implantations may be necessary to characterize these biases accurately enough to warrant changes in the methodology or hardware.

\section{Imaging of Distribution}

The advantage of real-time imaging of drug distribution includes the ability to accurately track infusate volume of distribution and troubleshoot delivery problems. A surrogate tracer method for MRI has been developed for CED that coinfuses Gd-DTPA (469 mg/ml, Magnevist) with the therapeutic agent. ${ }^{6}$ As such, iMRI will be increasingly used for monitoring drug delivery and preventing poor and ineffective distribution of the infusate..$^{15}$ The iMRI-guided trajectory frame used in this study is an integrated platform that provides accurate targeting and is compatible with real-time monitoring of CED infusate.

\section{Potential Advantages of Real-Time iMRI Cannula Placement}

Using conventional stereotactic (frame-based and frameless) methods, CED cannula placement is performed based on preoperative imaging. ${ }^{3,9,15}$ During cannula placement, significant shifts in the position of cortical and subcortical structures correlating with the amount of pneumocephalus can occur. ${ }^{5}$ There are several advantages to using an iMRI-based system for cannula placement. First, imaging during cannula placement permits assessment of cannula placement/trajectory accuracy in real time that allows for contemporaneous adjustments. Second, the use of an iMRI system for cannula placement allows for evaluation of significant shift of subcortical structures before and during infusion, which permits adjustment to planning. ${ }^{12}$ Similarly, in patients requiring multiple infusions, an iMRI targeting platform can identify brain shift during or caused by previous CED infusions and potential readjustment. Third, an integrated iMRI platform allows the surgeon to perform the entire procedure, including planning, insertion, and monitoring of infusate, with maximal efficiency. Fourth, in patients with planned multiple infusions, bilateral cannulae can be placed through the use of 2 simultaneously placed frames. Multiple ipsilateral sites can be targeted simply by changing the trajectory of a unilaterally placed guide frame, while leaving the base undisturbed. Finally, this system allows for adaptive planning and insertion of additional catheters if inadequate coverage of the target region was detected during a single setting. Currently available (frame-based and frameless) stereotactic systems, in the absence of updated MRI during cannula placement and infusion, have limited flexibility based on static preoperative imaging.

\section{Potential Limitations}

Consistent with any new technology, there are potential limitations. First, while iMRI is an intuitive system to use, there is a learning curve associated with using it. Use of the system may initially take more time than a routinely applied and more familiar frame-based or frameless stereotactic apparatus. However, with increased use, this technology should enhance the speed of cannula placement compared with other available methods. Second, this system requires use of an MR scanner in an operative environment for placement of the cannula(e). Nevertheless, there are significant advantages to real-time imaging during placement of infusion cannula(e), including the ability to contemporaneously correct the cannula trajectory and placement. Finally, further application of this technology for cannula placement in a larger number of patients will be necessary to determine its ultimate accuracy under a variety of pathological conditions.

\section{Conclusions}

Intraoperative MRI-based targeting and CED cannula placement is a safe and accurate technique that can be used 
in both supratentorial and infratentorial locations. Errors in targeting were minimal and permitted selective perfusion of brain pathology for pharmacological manipulation.

\section{Acknowledgments}

This study was funded by the intramural research program of the National Institute of Neurological Disorders and Stroke (NINDS) at the National Institutes of Health. IL13-PE was provided by InSys Therapeutics, Inc., under a cooperative research and development agreement with NINDS. The SmartFlow cannula and ClearPoint system were provided by MRI Interventions, Inc., under a clinical trial agreement with NINDS.

\section{References}

1. Asthagiri AR, Walbridge S, Heiss JD, Lonser RR: Effect of concentration on the accuracy of convective imaging distribution of a gadolinium-based surrogate tracer. J Neurosurg 115:467-473, 2011

2. Barua NU, Miners JS, Bienemann AS, Wyatt MJ, Welser K, Tabor AB, et al: Convection-enhanced delivery of neprilysin: a novel amyloid- $\beta$-degrading therapeutic strategy. J Alzheimers Dis 32:43-56, 2012

3. Cachón-González MB, Wang SZ, McNair R, Bradley J, Lunn D, Ziegler R, et al: Gene transfer corrects acute GM2 gangliosidosis-potential therapeutic contribution of perivascular enzyme flow. Mol Ther 20:1489-1500, 2012

4. Chen PY, Ozawa T, Drummond DC, Kalra A, Fitzgerald JB, Kirpotin DB, et al: Comparing routes of delivery for nanoliposomal irinotecan shows superior anti-tumor activity of local administration in treating intracranial glioblastoma xenografts. Neuro Oncol 15:189-197, 2013

5. Elias WJ, Fu KM, Frysinger RC: Cortical and subcortical brain shift during stereotactic procedures. J Neurosurg 107:983-988, 2007

6. Floyd DH, Kefas B, Seleverstov O, Mykhaylyk O, Dominguez $\mathrm{C}$, Comeau L, et al: Alpha-secretase inhibition reduces human glioblastoma stem cell growth in vitro and in vivo by inhibiting Notch. Neuro Oncol 14:1215-1226, 2012

7. Hdeib A, Sloan A: Targeted radioimmunotherapy: the role of ${ }^{131} \mathrm{I}$-chTNT-1/B mAb (Cotara) for treatment of high-grade gliomas. Future Oncol 8:659-669, 2012

8. Huo T, Barth RF, Yang W, Nakkula RJ, Koynova R, Tenchov $\mathrm{B}$, et al: Preparation, biodistribution and neurotoxicity of liposomal cisplatin following convection enhanced delivery in normal and F98 glioma bearing rats. PLoS ONE 7:e48752, 2012

9. Kunwar S, Chang S, Westphal M, Vogelbaum M, Sampson J, Barnett G, et al: Phase III randomized trial of CED of IL13-PE38QQR vs Gliadel wafers for recurrent glioblastoma. Neuro Oncol 12:871-881, 2010

10. Larson PS, Starr PA, Bates G, Tansey L, Richardson RM, Martin AJ: An optimized system for interventional magnetic resonance imaging-guided stereotactic surgery: preliminary evaluation of targeting accuracy. Neurosurgery 70 (1 Suppl Operative):95-103, 2012
11. Lonser RR, Corthésy ME, Morrison PF, Gogate N, Oldfield EH: Convection-enhanced selective excitotoxic ablation of the neurons of the globus pallidus internus for treatment of parkinsonism in nonhuman primates. J Neurosurg 91:294302, 1999

12. Pugh JA, Cox AG, McLeod CW, Bunch J, Writer MJ, Hart SL, et al: Elemental imaging of MRI contrast agents: benchmarking of LA-ICP-MS to MRI. Anal Bioanal Chem 403:1641-1649, 2012

13. Richardson RM, Kells AP, Martin AJ, Larson PS, Starr PA, Piferi PG, et al: Novel platform for MRI-guided convectionenhanced delivery of therapeutics: preclinical validation in nonhuman primate brain. Stereotact Funct Neurosurg 89:141-151, 2011

14. Richardson RM, Kells AP, Rosenbluth KH, Salegio EA, Fiandaca MS, Larson PS, et al: Interventional MRI-guided putaminal delivery of AAV2-GDNF for a planned clinical trial in Parkinson's disease. Mol Ther 19:1048-1057, 2011

15. Sampson JH, Archer G, Pedain C, Wembacher-Schröder E, Westphal M, Kunwar S, et al: Poor drug distribution as a possible explanation for the results of the PRECISE trial. J Neurosurg 113:301-309, 2010

16. White E, Bienemann A, Pugh J, Castrique E, Wyatt M, Taylor H, et al: An evaluation of the safety and feasibility of convection-enhanced delivery of carboplatin into the white matter as a potential treatment for high-grade glioma. J Neurooncol 108:77-88, 2012

17. Wood JD, Lonser RR, Gogate N, Morrison PF, Oldfield EH: Convective delivery of macromolecules into the naive and traumatized spinal cords of rats. J Neurosurg 90 (1 Suppl):115-120, 1999

18. Writer MJ, Kyrtatos PG, Bienemann AS, Pugh JA, Lowe AS, Villegas-Llerena C, et al: Lipid peptide nanocomplexes for gene delivery and magnetic resonance imaging in the brain. $\mathbf{J}$ Control Release 162:340-348, 2012

19. Yin D, Valles FE, Fiandaca MS, Bringas J, Gimenez F, Berger MS, et al: Optimal region of the putamen for image-guided convection-enhanced delivery of therapeutics in human and non-human primates. Neuroimage 54 (Suppl 1):S196-S203, 2011

\section{Author Contributions}

Conception and design: Lonser, Heiss. Acquisition of data: Lonser, Heiss. Analysis and interpretation of data: Chittiboina. Drafting the article: Chittiboina. Critically revising the article: all authors. Reviewed submitted version of manuscript: all authors. Approved the final version of the manuscript on behalf of all authors: Lonser. Study supervision: Lonser, Heiss.

\section{Correspondence}

Russell R. Lonser, Department of Neurological Surgery, The Ohio State University Wexner Medical Center, 410 W. 10th Ave., N1047 Doan Hall, Columbus, OH 43210. email: russell. lonser@osumc.edu. 